\title{
OPTIMAL EMERGENCY VEHICLE BRAKING CONTROL BASED ON DYNAMIC FRICTION MODEL
}

\author{
L. Olmos \& L. Álvarez-Icaza
}

Instituto de Ingeniería

Universidad Nacional Autónoma de México

04510, Coyoacán DF, México

LOImosG@iingen.unam.mx

alvar@pumas.iingen.unam.mx

Received: January $20^{\text {th }}$ 2003. Accepted March $30^{\text {th }} 2005$

\section{ABSTRACT}

A dynamic friction model for the tire-road interface is used in an optimal control scheme for emergency braking of vehicles. The controller sets a target relative velocity curve that the vehicle must track in order to achieve braking in minimum time. It is shown that this curve corresponds to the solution of a minimum time optimal control problem. The final goal of the control is to improve the safety levels in highway transportation.

\section{RESUMEN}

Se utiliza un modelo dinámico de fricción para la interfase llanta-pavimento en el diseño de un esquema de control óptimo para el frenado de emergencia de vehículos. El controlador establece una curva de referencia para la velocidad relativa que el vehículo debe seguir para poder alcanzar el frenado en tiempo mínimo. Se muestra que esta curva corresponde con la solución de un problema de control óptimo. El objetivo final de este esquema de control es aumentar los niveles de seguridad en autopistas.

KEYWORDS: Optimal control, Vehicular control, Dynamic friction model.

\section{INTRODUCTION}

The main purpose for the increasing levels of automation in vehicles is to improve the capacity of highway systems and to sustain or improve its actual safety levels. One of the key points that is necessary to achieve safe operation of these systems lies in their ability to perform emergency braking maneuvers properly, as according to [1] the most drastic faults in automated highways imply performing this kind of manuever. Daily situations indicate that this is also the case for manual driving. Safety during emergency braking is very much related with the braking capability of each vehicle. When this capability is known, it is possible to set appropriate minimum intervehicle distances that will preserve safety during emergency braking [2]. Braking capability depends mostly on the tire-road contact forces. These are difficult to know precisely because they change with the type of tire, road and weather conditions.

The goal of this paper is to present the design of an optimal emergency braking control. The design uses a dynamical friction model to describe the interaction forces between tire and road and assumes that the parameters of the model are known or that is possible to estimate them with some adaptive scheme [3]. This dynamic friction model applied to tire-road contact was first introduced by [16]. In this paper, however, the emphasis is not on deriving the instantaneous tire-road contact force. The goal is rather to obtain a global description of the behaviour of this force and, based on it, designing a target surface that the controller must track during emergency braking in order to 
achieve minimum time braking. This controller is intended for use in a warning system that would allow vehicles to keep always a safe distance with respect to vehicle in front.

Using a singular perturbations analysis approach it is possible to prove that the optimal braking trajectory can be computed with the velocity of the vehicle and the relative velocity between tire and road'.

The paper is divided into seven sections, including this introduction. Section 2 presents a simplified model of the vehicle dynamics, while section 3 describes the first order dynamic friction model that describes the tire-road interface. In section 4 the optimal control analysis is presented and section 5 presents an alternative design controller using Lyapunov techniques. Simulation results are described in section 6 and, finally, section 7 presents conclusions and directions for future work.

\section{VEHICLE MODELING}

In this paper only the longitudinal dynamic is considered, a quarter vehicle model is used ${ }^{2}$. The longitudinal motion of the vehicle is expressed by

$$
m \dot{v}=4 F_{x}-F_{a x},
$$

where $v$ is the vehicle longitudinal velocity, $m$ is its mass, $F_{x}$ is the tire-road force and $F_{a x}$ is the aerodynamic drag force. This equation assumes that the forces in the tires are evenly distributed

The rotational dynamics of the wheel is described by

$$
I \dot{\omega}=\tau_{d}-\tau_{b}-F_{x} R
$$

where $\omega$ is the angular velocity, / the moment of inertia of the wheel, $\tau_{d}$ is the driving torque, $\tau_{b}$ is the braking torque and $R$ is the effective radius of the wheel. In Eqs. (1) and (2), it is assumed that the longitudinal velocity $v$ and the angular velocity are related by the relative velocity $s$ defined as

$$
s=v-R \omega .
$$

The time derivate of Eq. (3) is

$$
\dot{s}=\dot{v}-R \dot{\omega}
$$

Substituting Eqs. (1) and (2) in Eq. (4)

$$
\dot{s}=-\frac{R}{I} \tau_{d}+\frac{R}{I} \tau_{b}+\frac{R^{2}}{I} F_{\chi}+\frac{4 F_{x}}{m}-\frac{F_{a x}}{m} .
$$

\footnotetext{
1 This is the difference between the velocity of the vehicle's centre of mass velocity and the tangential velocity of the tire at the zone of contact with the road.

${ }^{2}$ This model is widely used in the literature; see [4, 5 and 6].
} 
The aerodynamic drag force $F_{a x}$ is usually expressed as

$$
F_{a x}=C_{a x} v^{2},
$$

with $C_{a x}$ a constant that depends on the effective longitudinal area, air density and the shape of the vehicle [7].

The force in the tire has negative direction during braking and can be expressed as a function of normal load and the friction coefficient

$$
F_{x}=-\mu F_{N}=-\mu \frac{m g}{4}
$$

where $\mu$ is the friction coefficient between tire and road and $F_{N}=m g / 4$ is the normal force in each tire. In this paper a plane road is considered, if the road has a slope, the normal load can be modified accordingly.

Substituting Eqs. (6) and (7) in Eqs. (1) and (5) vehicle dynamics is described by

$$
\begin{gathered}
\dot{v}=-c \mu-d v^{2}, \\
\dot{s}=-(a+c) \mu-b-d v^{2}+e \tau_{b},
\end{gathered}
$$

with $a=R^{2} m g / 4 I, b=R \tau_{d} / I, c=g, d=C_{a x} / m$ and $e=R / I$. Finally the braking torque $\tau_{b}$ can be expressed as $\tau_{b}=K_{b} P_{b}$, with $K_{b}$ a known constant and $P_{b}$ the pressure in the master cylinder, that is the control signal.

\section{TIRE-ROAD FRICTION INTERFACE}

Most of the literature related to tire-road friction is based on pseudo-static models for friction as in [8] and [9] in which friction coefficient $\mu$ is a function of the tire $\operatorname{slip}^{3} \rho$ and some other parameters like vehicle velocity and normal load. Recently, Canudas et al [10] introduced dynamic friction models that include one or more internal states, looking for a better description of the friction phenomenon. These new dynamical models are based on the idea that the surfaces in contact are very irregular at a microscopic level and therefore the contact occurs at different points between these irregularities. This contact can be seen in a simple way like the one that happens between elastic bristles and that is illustrated in Fig. 1. When a tangent force is applied these bristles are bent like springs, increasing the friction force, if the force is large enough, these bristles will deform in such a way that they will slip. Obviously this is a random phenomenon because of the irregularity of the surfaces.

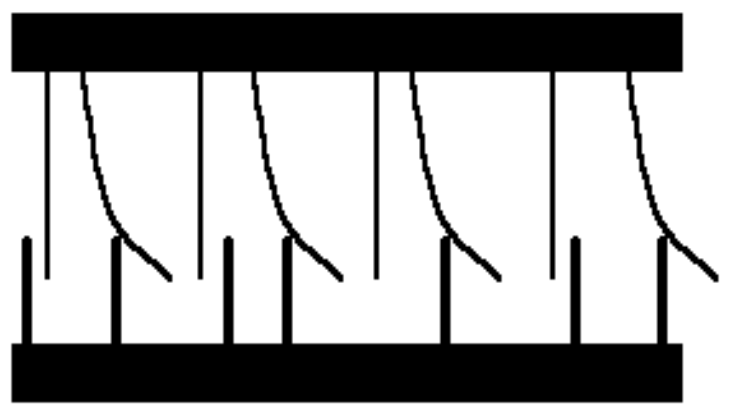

Figure 1. Interface between two surfaces through elastic bristles

${ }^{3}$ Slip $\rho$ is defined as $\rho=s / v$. 
The model presented here uses the average deformation of the bristles $z$ and is similar to the one used in [11] and is of the form

$$
\begin{gathered}
\frac{d z}{d t}=s-\frac{\sigma_{0}|s|}{g(s)} z, \\
\mu=\frac{F}{F_{n}}=\sigma_{0} z+\sigma_{1} \dot{z}-\sigma_{2} s, \\
g(s)=\mu_{c}+\left(\mu_{c}-\mu_{c}\right) e^{-\left|\frac{s}{v_{s}}\right|^{1 / 2}}
\end{gathered}
$$

where $\sigma_{0}$ is the normalized longitudinal stiffness, $\sigma_{1}$ the normalized longitudinal damping and $\sigma_{2}$ is the normalized viscous relative damping. These three parameters characterize a specific road type. The function $g(s)$ intents to model the Stribeck effect [10]; $\mu_{c}$ is the normalized Coulomb friction, $\mu_{s}$ is the normalized static friction $\left(\mu_{c} \leq \mu_{s} \in[0,1]\right), v_{s}$ is the Stribeck relative velocity. This last parameter plays the role of a time constant of the system, when $g(s)$ is considered as a first order system.

When the new dynamic model of friction is introduced, the order of the complete system increases. However, the inner dynamics of the friction state $z$ is much faster than that of $s$ and $v$. This fact was proven using a singular perturbation approach in [12] $]^{4}$. Based on this approach, it is possible, from Eqs. (10) and (12), to obtain the equilibrium of the internal state in terms of $s$ and $\rho$, that is

$$
z=\frac{g(s)}{\sigma_{0}}\left(1-e^{-\frac{\sigma_{0}|\rho|}{g(s)} L}\right)
$$

When Eq. (13) is substituted in Eq. (11), this results in a friction function of the form

$$
\mu=g(s)+\left(\sigma_{1} s-g(s)\right) e^{-\frac{\sigma_{0}|s| L}{g(s) v}}-\sigma_{2} s
$$

This last equation represents a pseudo-static solution to the dynamic friction model that is comparable to those in [8] and [9]. By using it, it is possible to compare in similar conditions pseudo-static and dynamic friction models. It is interesting to remark that, although the equivalent pseudo-static solution in Eq. (14) was derived directly from a dynamic friction model, it has an analytic structure with some points in common with the pseudo-static model presented in [9] that was derived solely based on experiments.

\footnotetext{
${ }^{4}$ See Theorem 9.1 in [13] for details about the singular perturbation analysis approach.

${ }^{5}$ More details in [12].
} 


\section{MINIMUM BRAKING TIME OPTIMAL CONTROL LAW DESIGN}

In optimal control theory the goal is to minimize a performance index of the system, this index is usually expressed in the form

$$
J=\int_{0}^{t_{f}} L d t .
$$

In this paper the goal is to minimize the time taken in an emergency braking, therefore $L=1$ and $J=t_{f}$ and the boundary conditions are $v_{0}=v_{0}, v_{f}=0, s_{o}=0$ and $s_{f}=0$ (see [14] for more details).

Taking the vehicle model in Eqs. (8) and (9) the Hamiltonian of the system is given by

$$
H=\lambda_{v}\left(-c \mu-d v^{2}\right)+\lambda_{s}\left(-(a+c) \mu-b-d v^{2}+e K_{b} P_{b}\right)+1
$$

and the adjoin system is

$$
\begin{gathered}
\dot{\lambda}_{v}=\lambda_{v}\left(c \frac{\partial \mu}{\partial v}+2 d v\right)+\lambda_{s}\left((a+c) \frac{\partial \mu}{\partial v}+2 d v\right), \\
\dot{\lambda}_{s}=\lambda_{v} c \frac{\partial \mu}{\partial s}+\lambda_{s}(a+c) \frac{\partial \mu}{\partial s} .
\end{gathered}
$$

Due to the fact that the final time is not specified, the transversality condition can be set as $H\left(t_{f}\right)=0$. Furthermore, in this case, the Hamiltonian is not an explicit function of time, therefore

$$
H(t)=0, \forall t \in\left[0, t_{f}\right]
$$

along the optimal trajectory. The optimal control is then given by

$$
P_{b_{\text {opt }}}=\arg \min H\left(x, \lambda, P_{b}\right)
$$

From Eq. (15), the switching function is

$$
H_{1}=\frac{\partial H}{\partial P_{b}}=\lambda_{s} e K_{b}
$$

Eq. (20) and its time derivative must vanish when the trajectories lie on a singularity of Eq. (20). Taking time derivative of Eq. (20) and substituting $\dot{\lambda}_{s}$

$$
\begin{gathered}
\dot{H}_{1}=e K_{b} \lambda_{s} \\
\dot{H}_{1}=e K_{b}\left[\lambda_{v} c \frac{\partial \mu}{\partial s}+\lambda_{s}(a+c) \frac{\partial \mu}{\partial s}\right] .
\end{gathered}
$$

The singular case of interest corresponds to the arc when $H_{1} \equiv 0$, which implies $\lambda_{s} \equiv 0$; substituting this condition yields 


$$
\dot{H}_{1}=e K_{b}\left[\lambda_{v} c \frac{\partial \mu}{\partial s}\right]=0
$$

Eq. (23) is very important because it shows that in the optimal trajectory $\frac{\partial \mu}{\partial s}=0$ or $\lambda_{v}=0$. If the second condition holds, the adjoin vector would be zero in the singular sub-arc. This is a contradiction because the adjoin vector must not vanish under any circumstance ${ }^{6}$. Therefore, $\frac{\partial \mu}{\partial s}=0$ along the singular sub-arc, regardless of $v$, the other state of the system.

It is possible to find the optimal control, demanding consistency in $H_{1} \equiv 0$ and $\cdot \dot{H}_{1} \equiv 0$. On the other hand, the control signal is physically bounded, i.e., $0 \leq P_{b} \leq P_{b \max }$. Therefore, analyzing Eqs. (19), (22) and (23) and the bounds on the control signal, $P_{\mathrm{bopt}}$ is given by the following law

$$
P_{b o p t}\left\{\begin{array}{lll}
P_{b_{\max }} & \text { if } & \dot{H}_{1}>0 \\
0 & \text { if } & \dot{H}_{1}<0 \\
P_{b_{\sin g}} & \text { if } & \dot{H}_{1} \equiv 0
\end{array} .\right.
$$

To guarantee $\dot{H}_{1}=0$ and to find $P_{b_{\text {sin } g}}$, it is necessary to take the second derivative of $H_{1}$ with respect time and substitute $\dot{\lambda}_{n}$ in it

$$
\ddot{H}_{1}=e c K_{b}\left\{\left[\lambda_{v}\left(c \frac{\partial \mu}{\partial v}+2 d v\right)+\lambda_{s}\left((a+c) \frac{\partial \mu}{\partial v}+2 d v\right)\right] \frac{\partial \mu}{\partial s}+\lambda_{v} \frac{d}{d t}\left(\frac{\partial \mu}{\partial s}\right)\right\} .
$$

Based on Eq. (23), the analysis reduces to find

$$
\ddot{H}_{1}=\frac{d}{d t}\left(\frac{\partial \mu}{\partial s}\right)=0
$$

To hold the Eq. (26), it is necessary that

$$
\begin{aligned}
\frac{d}{d t}\left(\frac{\partial \mu}{\partial s}\right) & =\frac{\partial^{2} \mu}{\partial s^{2}} s+\frac{\partial^{2} \mu}{\partial v \partial s}=0 \\
& =\frac{\partial^{2} \mu}{\partial s^{2}}\left(-(a+c) \mu-b-d v^{2}+e K_{b} P_{b}\right)+\frac{\partial^{2} \mu}{\partial v \partial s}\left(-c \mu-d v^{2}\right)=0
\end{aligned} .
$$

After some manipulations to Eq. (27), the singular control $P_{b_{\text {sing }}}$ is

$$
P_{b \sin g}=\frac{1}{e K b}\left[\frac{\partial^{2} \mu}{\partial v \partial s}\left(c \mu+d v^{2}\right)\left(\frac{\partial^{2} \mu}{\partial s^{2}}\right)^{-1}+(a+c) \mu+d v^{2}\right] .
$$

${ }^{6}$ See $[15]$. 


\section{LYAPUNOV BASED, MAXIMUM FRICTION CONTROL LAW}

This section presents a design that is based on the results derived in the previous section to obtain the optimal control, that is, however, easier to implement. The goal of this controller is to force the system state into a desired a reference trajectory. This trajectory is represented by a desired relative velocity $s_{d}$ that produces maximum friction force at the current vehicle velocity and will lead to zero velocity at the end of the emergency braking process.

Using Eq. (23), that states $\left(\frac{\partial \mu}{\partial s}\right)=0$ and Eq. (14)

$$
\frac{\partial \mu}{\partial s}=g^{\prime}(s)+e^{-\frac{\sigma_{0} f(s) L}{v}}\left[\left(\sigma_{1}-g^{\prime}(s)\right)-\frac{\sigma_{0} f^{\prime}(s) L}{v}\left(\sigma_{1} s-g(s)\right)\right]=0,
$$

where

$$
g^{\prime}(s)=\frac{\partial g(s)}{\partial s} \quad f^{\prime}(s)=\frac{\partial f(s)}{\partial s}
$$

For a given value of $v$, Eq. (29) allows to obtain a desired value for the relative velocity $s_{d}$ such that Eq. (23) is satisfied ${ }^{7}$. The next step in this controller design is trying to take the system state to this curve. For this purpose, the following error signal is introduced

$$
\bar{s}=s-s_{d} .
$$

Whose time derivative is

$$
\dot{\bar{s}}=\dot{S}-\dot{S}_{d}
$$

Define the Lyapunov function candidate

$$
W=\frac{1}{2} \bar{s}^{2}+\frac{1}{2} v^{2}+\frac{K}{2}\left(\int \bar{s} d t\right)^{2}
$$

with $K>0$. Taking the time derivative of Eq. (32) and substituting Eqs. (8), (9) and (31)

$$
\begin{aligned}
\dot{W} & =\bar{s} \bar{s}+K \bar{s} \int \bar{s} d t \\
& =\bar{s}\left[-(a+c) \mu-b-d v^{2}+e K_{b} P_{b}-s_{d}\right]+v\left[-c \mu-d v^{2}\right]+K \bar{s} \int \bar{s} d t
\end{aligned}
$$

If the control signal $P_{b}$ is taken as

$$
P_{b}=\frac{1}{e K_{b}}\left[(a+c) \mu+d \mu^{2}+H \bar{s}+K_{1} \int \bar{s} d t\right]
$$

and substituted into Eq. (33), then

$$
\dot{W}=-c \mu v-d v^{3}+H \bar{s}^{2}+\left(K_{1}+K\right) \bar{s} \int \bar{s} d t
$$

${ }^{7}$ See [12] for details. 
Analyzing this last equation, considering that $v$ and $\mu$ are positive at every time and that $c$ and $d$ are positive constants, if $K_{i}$ is chosen in such a way that the last term is negative and $H<0$, then Eq. (35) becomes negative definite.

Due to the fact that $\int \bar{s} d t$ is only zero when $\bar{s} \equiv 0$; and using Barbalat's lemma, it can be concluded that the point $\bar{s}=0$ and $v=0$ is asymptotically stable.

\section{SIMULATIONS RESULTS}

In this section simulation results of an emergency braking maneuver are presented for both control laws described in the previous sections. The parameters used for the vehicle model were borrowed from [16] and are shown in Table I. Prior to attempting the emergency braking manoeuvre, vehicles are cruising at a constant speed of $30 \mathrm{~m} / \mathrm{s}$.

Table / Simulation parameters

\begin{tabular}{|l|l|}
\hline Vehicle mass, $m$ & $1701[\mathrm{~kg}]$ \\
\hline Wheel inertia, $I$ & $2.603[\mathrm{~kg} \mathrm{~m}]$ \\
\hline Wheel radius, $R$ & $0.323[\mathrm{~m}]$ \\
\hline Brake system gain, $K_{b}$ & $0.9\left[\mathrm{~m}^{2}\right]$ \\
\hline Aerodynamic drag constant, $C_{a x}$ & $0.3693[\mathrm{~kg} / \mathrm{m}]$ \\
\hline Longitudinal stiffness, $\sigma_{0}$ & $40[1 / \mathrm{m}]$ \\
\hline Longitudinal damping, $\sigma_{1}$ & $0.0049[\mathrm{~s} / \mathrm{m}]$ \\
\hline Viscous relative damping, $\sigma_{2}$ & $0.0018[\mathrm{~s} / \mathrm{m}]$ \\
\hline Normalized Coulomb friction coefficient, $\mu_{c}$ & 0.6 \\
\hline Normalized static friction coefficient, $\mu_{s}$ & 0.9 \\
\hline Stribeck velocity, $v_{s}$ & $12.5[\mathrm{~m} / \mathrm{s}]$ \\
\hline
\end{tabular}

Fig. 2 shows the evolution of the system states, $v$ and $s$, when the optimal control law is applied. It is clear that the velocity decreases rapidly to zero, from its initial value of $30 \mathrm{~lm} / \mathrm{s}]$. In Fig. 3, the deceleration attained during the braking process and the braking torque are shown. From the pressure plot it can be seen that initially the system applies maximum pressure until the relative velocity is taken to the singular solution of the optimal control law. Once there, the pressure is controlled so as to keep the relative velocity in the singular arc, solution to the optimal control problem. Some switching to zero pressure can also be observed. The evolution of the Hamiltonian and its time derivative, $H_{1}$ and $\dot{H}_{1}$, are shown in Fig. 4 , where it is clear that their values are very close to zero, as expected. 

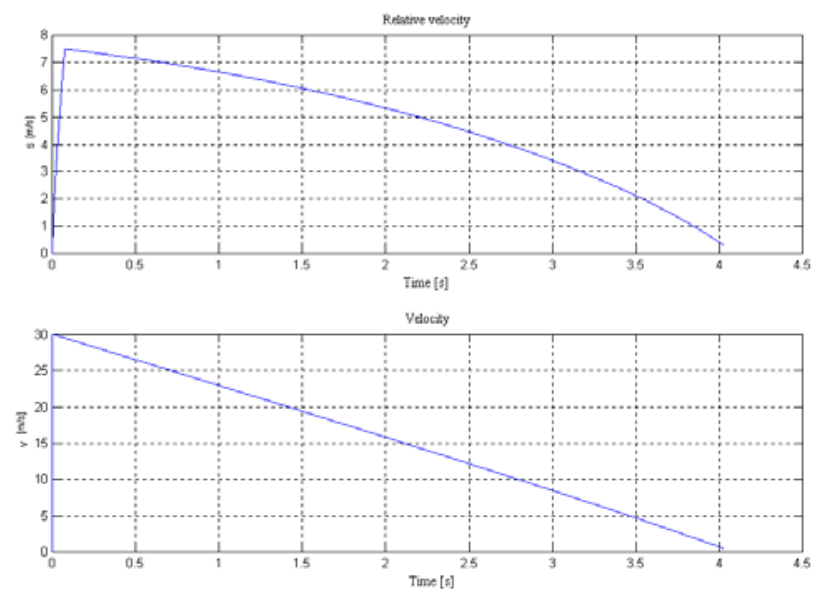

Figure 2. Minimum braking time control law; time evolution of the system states: velocity $v$ and relative velocitys
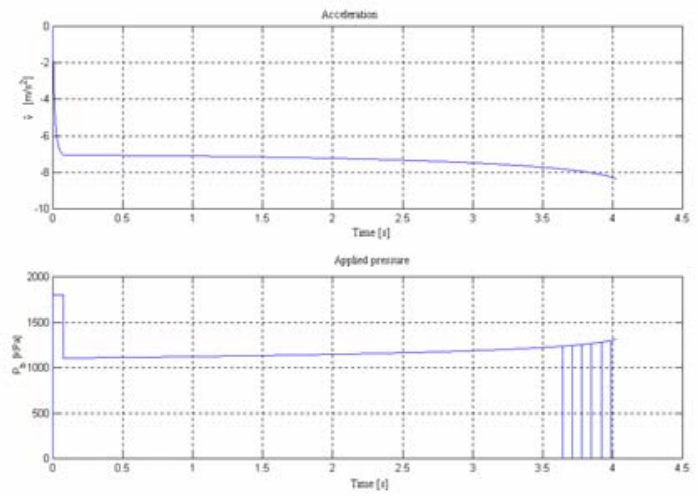

Figure 3. Minimum braking time control law; acceleration during braking and pressure in the master cylinder $P_{b}$
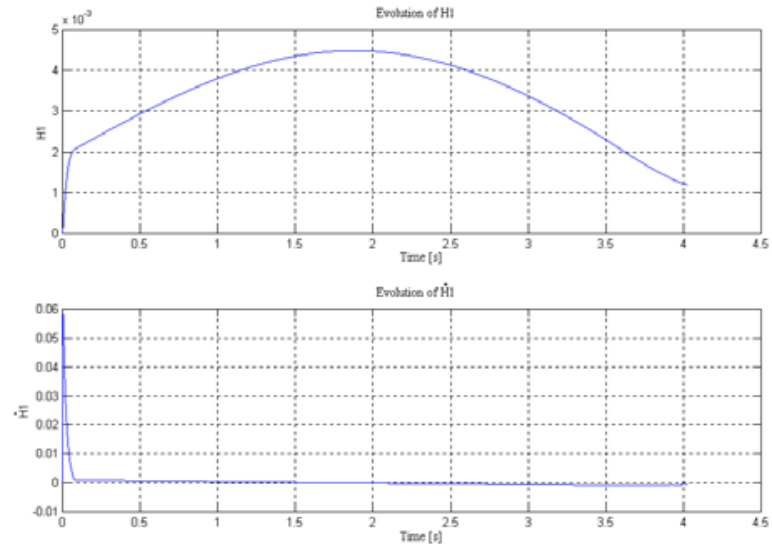

Figure 4. Minimum braking time control law; time evolution of switching function $H_{1}$ and its time derivative $H_{1}$ 
The simulation results when the maximum friction law is applied are shown in Figs. 5-7. Fig. 5 illustrates the time evolution of the desired and real relative velocities and their difference (left, right and bottom plot, respectively). Fig. 6 shows the velocity and deceleration in the braking process. It can be noted that, as friction increases with reduction in velocity, the deceleration is higher at low velocities. Finally, Fig. 7 shows the braking torque applied to the wheels and the coefficient of friction, both as function of time.
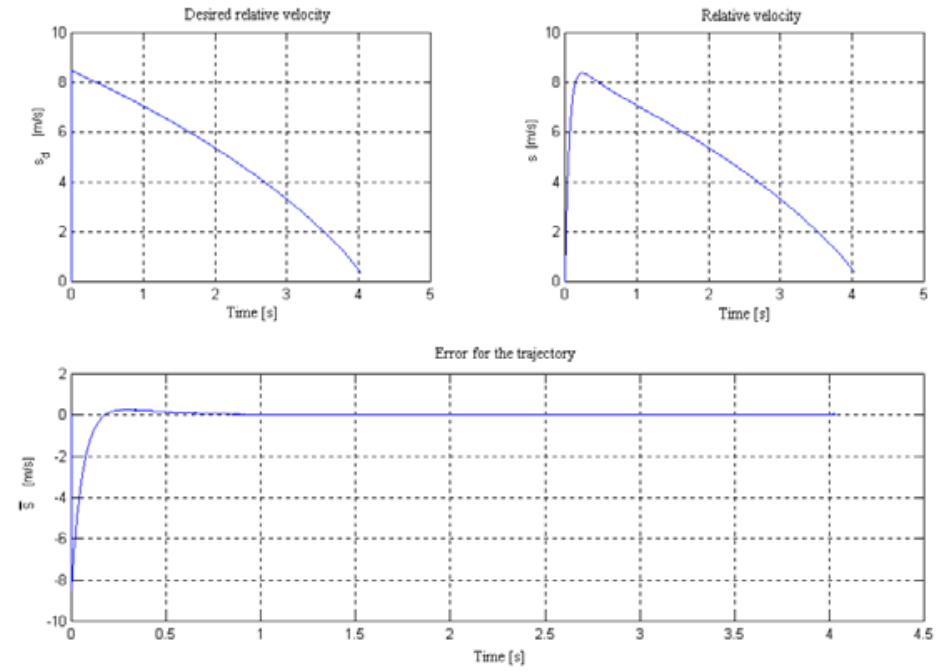

Figure 5. Lyapunov based maximum friction control law; Reference relative velocity $s_{d}$, real relative velocitys and error in relative velocity $\bar{s}$ during the braking process
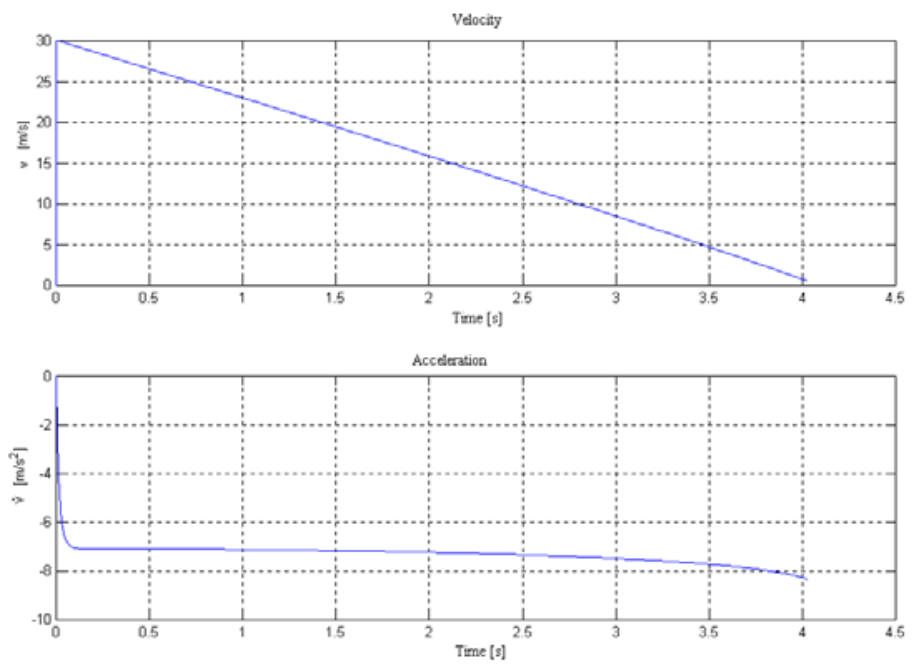

Figure 6. Lyapunov based maximum friction control law; Velocity v and acceleration during braking 

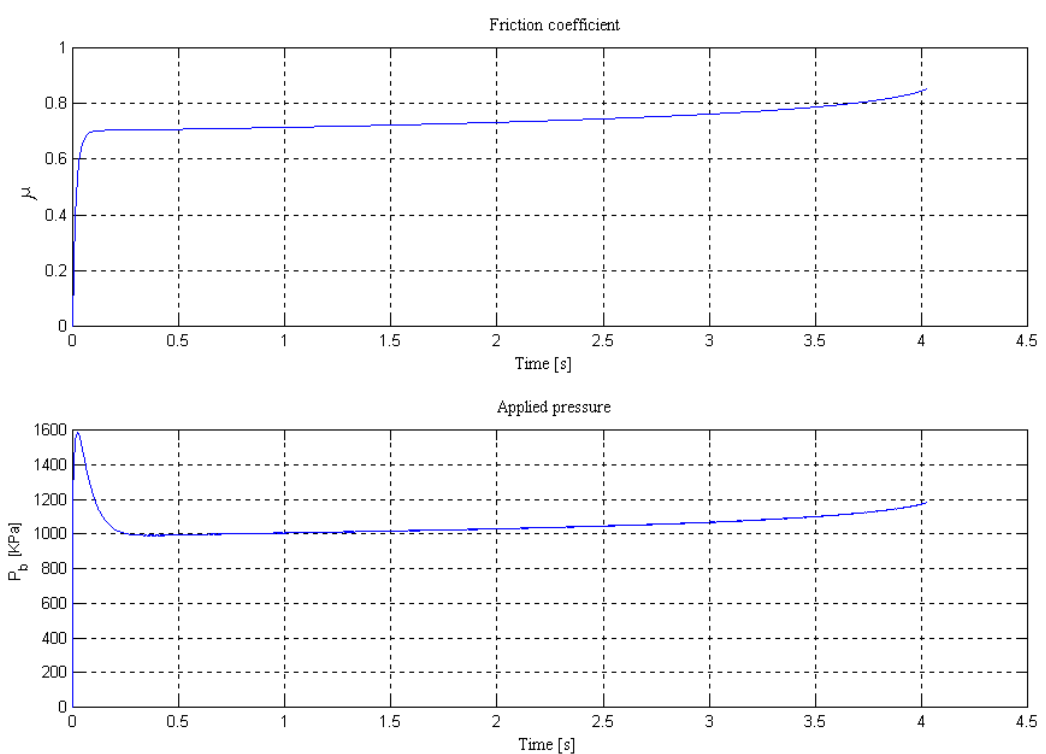

Figure 7. Lyapunov based maximum friction control law; Pressure in the master cylinder $P_{b}$ and friction coefficient $\mu$

To compare the two control laws, Fig. 8 was included. It shows the two master cylinder pressure signals, the optimal braking pressure, $P b_{\text {opt }}$ given by Eq. (24), and the maximum friction pressure, $P_{b}$, given by Eq. (34). From the plots, it is clear that both control laws are very similar and that the difference between the two signals is close to zero.
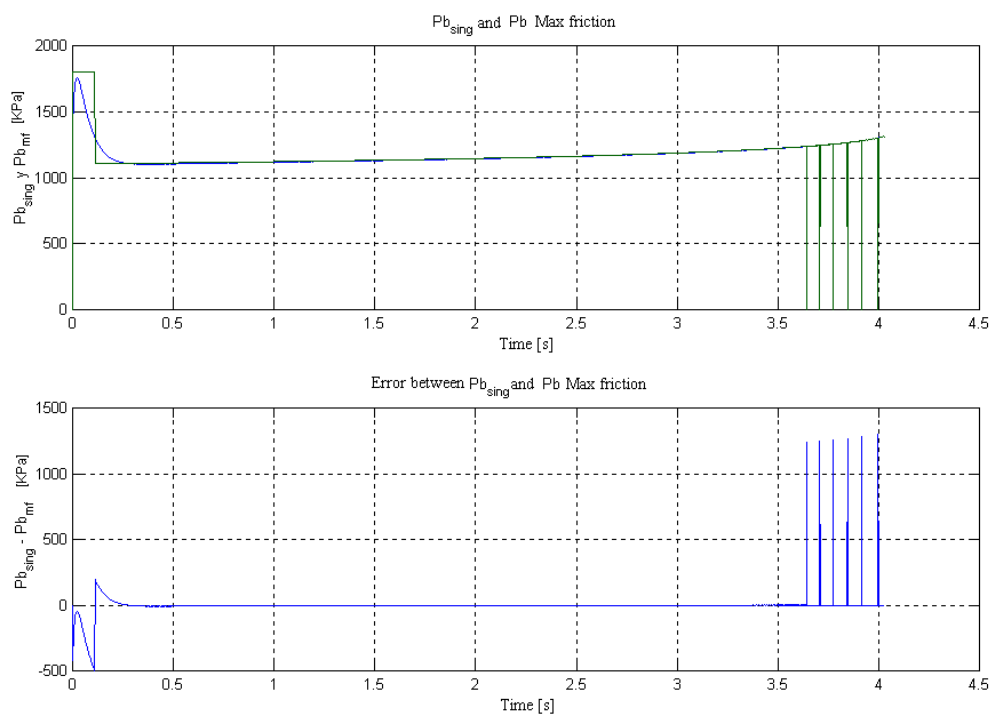

Figure 8. Comparison between minimum braking time control law and Lyapunov based maximum fricition control law and difference between the two control signals 


\section{CONCLUSION}

In this paper an emergency braking control law for wheeled vehicles was designed using optimal control theory. The design of this law is based on the assumption that the force at the tire-road interface can be appropriately described by a dynamic friction model. As expected in minimum time optimal control problems, the solution consist in keeping the master cylinder pressure in the curve that represents the minimization of the Hamiltonian. As a simpler alternative in terms of implementation, a maximum friction control law was designed based on Lyapunov techniques. Simulation results of an emergency braking maneuver using both control laws are presented. The performance in both cases is extremely good and only slight differences can be appreciated between the two control laws. This controller is intended for use in conjunction with a warning system that would allow drivers to keep always a safe distance with respect to vehicle in front.

\section{ACKNOWLEDGMENTS}

Research partially financed by CONACYT grant 31226U.

\section{REFERENCES}

[1] Lygeros, J. D., Godbole, N., and Broucke, M. E. "A fault tolerant control architecture for automated highway systems," IEEE Transactions on Control Systems Technology, vol. 8,pp. 205-219, 2000.

[2] L. Alvarez and R. Horowitz, "Safe platooning in AHS. Part I: safety regions design, "Vehicle System Dynamics, vol. 32, no. 1,pp. 23-56, 1999.

[3] J. Yi, L. Alvarez, X. Claeys, R. Horowitz, C. Canudas, and L. Olmos," Adaptive emergency braking control with observer-base dynamic tire/road friction model and underestimation of friction coefficient." Proceedings of the IFAC'02 World Congress, Paper \#2252, T-ThA02, 2002.

[4] U. Kiencke and A. Daiss, "Estimation of tyre friction for enhanced ABS-systems," in Proceedings of the AVEG'94, 1994.

[5] U. Kiencke, "Realtime estimation of adhesion characteristic between tyres and road," in Proceedings of the IFAC World Congress, vol. 1, pp. 15-22, 1993.

[6] Y. Liu and J. Sun,"Target slip tracking using gain scheduling for anti-lock braking systems," in Proceedings of the 1995 American Control Conference, pp. 1178-1182, 1995.

[7] T. Gillespie, Fundamentals of Vehicle Dynamics. Warrendale, PA: Society of Automotive Engineers, Inc., 1992.

[8] E. Bakker, L. Nyborg and H. Pacejka, "Tyre modelling for use in vehicle dynamic studies." Society of Automotive Engineers Paper \# 870421, 1987.

[9] M. Burckhardt, Fahrwerktechnik: Radschlupfrege/systeme. Germany: Vogel-Verlag, 1993.

[10 ] C. Canudas de Wit, H. Olsson, K. J. 'Astrom, and P. Lischinsky, "A new model for control of systems with friction," IEEE Transactions on Automatic Control, vol. 40, no. 3, pp. 419-425, 1995.

[11] C. Canudas and R. Horowitz, "Observers for tire/road contact friction using only whell angular velocity information," in Proceedings of the 1999 Conference on Decision and Control, pp. 3932-3937, 1999.

[12] L. Olmos, "Control de frenado de emergencia con base en modelos de fricción llanta pavimento," Master's thesis, Programa de Maestría y Doctorado en Ingeniería, Universidad Nacional Autónoma de México, 2001.

[13] H. K. Khalil, Nonlinear Systems. USA: Prentice-Hall, second ed., 1996.

[14] A. E. Bryson and Y.-C. Ho, Applied Optimal Control. USA: Blaisdell Publishing Company, first ed., 1969.

[15] P. Tsiotras and C. Canudas, "On the optimal braking of wheeled vehicles," in Proceedings of the 2000 American Control Conference, pp. 569-573, 2000.

[16] C. Canudas and P. Tsiotras, "Dynamic tire friction models for vehicle traction control," in Proceedings of the 1999 Conference on Decision and Control, pp. 3746-3751, 1999. 